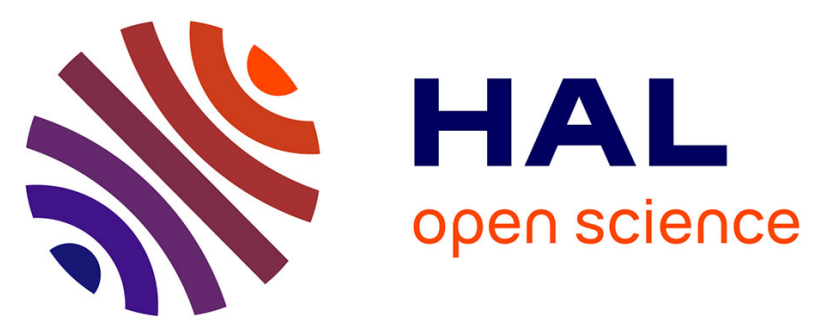

\title{
Enhanced Amplitude Noise Tolerance of a Self-Seeded RSOA Laser Using Balanced Detection
}

Mathilde Gay, Laurent Bramerie, Kamal Hussain, Thierry Chartier, Stephane Trebaol, Jean-Claude Simon, Anaelle Maho, Romain Brenot

\section{- To cite this version:}

Mathilde Gay, Laurent Bramerie, Kamal Hussain, Thierry Chartier, Stephane Trebaol, et al.. Enhanced Amplitude Noise Tolerance of a Self-Seeded RSOA Laser Using Balanced Detection. IEEE Photonics Technology Letters, 2017, 29 (24), pp.2219-2221. 10.1109/LPT.2017.2771400 . hal-01713943

\section{HAL Id: hal-01713943 \\ https://hal.science/hal-01713943}

Submitted on 21 Feb 2018

HAL is a multi-disciplinary open access archive for the deposit and dissemination of scientific research documents, whether they are published or not. The documents may come from teaching and research institutions in France or abroad, or from public or private research centers.
L'archive ouverte pluridisciplinaire $\mathbf{H A L}$, est destinée au dépôt et à la diffusion de documents scientifiques de niveau recherche, publiés ou non, émanant des établissements d'enseignement et de recherche français ou étrangers, des laboratoires publics ou privés. 


\title{
Enhanced Amplitude Noise Tolerance of a Self- Seeded RSOA Laser using Balanced Detection
}

\author{
Mathilde Gay, Laurent Bramerie, Kamal Hussain, Thierry Chartier, Stéphane Trebaol, \\ Jean-Claude Simon, Anaëlle Maho, and Romain Brenot
}

\begin{abstract}
Self-seeded cavity lasers appear as attractive colorless sources for dense wavelength division multiplexed passive optical networks. Although this technology has already demonstrated high performance in a number of experiments, the strong multimode behavior of these kilometer-long cavities results in large relative intensity noise (RIN) that can become the main limiting factor for transmission. In this letter, we correlate the transmission performance degradation to the RIN increase when the cavity length increases. We evidence a better tolerance to amplitude noise when a combination of Manchester encoding and balanced detection are used in a proof-of-concept experiment. Using this combination, the cavity length can be four times longer than when using standard non-return-to-zero encoding for the same bit-error-rate performance.
\end{abstract}

Index Terms - Reflective semiconductor optical amplifier, colorless source, DWDM Passive optical network, RIN.

\section{INTRODUCTION}

$\mathrm{R}$ EFLECTIVE semiconductor optical amplifier (RSOA) fiber self-seeded cavity lasers have been demonstrated as attractive colorless sources for dense wavelength division multiplexed (WDM) passive optical networks (PONs). A key advantage is that they provide passive tuning of the wavelength. This simply results from the selection of a given port of the wavelength multiplexer, which can be several kilometers away from the RSOA [1-5]. This contrasts with the use of singlemode semiconductor lasers, which need to be designed for a pre-set emission frequency and require active tuning and frequency control. This in turn eases the network management and reduces inventory costs. Furthermore, these sources provide a wide optical bandwidth (more than $60 \mathrm{~nm}$ ). The RSOA, which has been shown to be compatible with uncooled operation [6], is used both for amplification and modulation. Many demonstrations of RSOA self-seeded cavities have been published so far, with bit rates up to $10 \mathrm{Gbit} / \mathrm{s}$ in the telecommunication $\mathrm{C}$-band and transmission distances up to $90 \mathrm{~km}$ in the O-band [1-3].

The behavior of these kilometer-long cavities has also been studied theoretically and experimentally $[4,5]$. The resulting

Manuscript received September 12 $2^{\text {th }}$, 2017. This work was supported in part by the French National Research Agency (ANR) under the LAMPION (ANR13-INFR-0002) project.

M. Gay, L. Bramerie, K. Hussain, T. Chartier, S. Trebaol, J.-C. Simon are with Foton Laboratory, CNRS, University of Rennes 1, Enssat, F-22300 Lannion, France (e-mail: mathilde.gay@enssat.fr). large number of longitudinal modes induces high relative intensity noise (RIN) dominated by mode beating after detection, which can become the main limiting factor for transmission, notably under the effect of chromatic dispersion $[4,5,7]$.

In this work we propose and investigate the use of balanced detection to overcome this limitation as it is known to reduce the impact of RIN [8, 9]. Such a detection scheme is however not compatible with simple non-return-to-zero (NRZ) encoded intensity modulation (IM) commonly employed in access networks together with direct detection. Manchester encoding is therefore employed instead since it is fully compatible with balanced detection $[9,10]$. RIN measurements confirm the impact of cavity length on intensity noise, in particular at low frequencies. These measurements are then correlated to the transmission performance degradation. Finally, an enhanced tolerance to cavity length of a self-seeded RSOA fiber cavity laser is demonstrated in a proof-of-concept experiment. Using balanced detection, a better eye opening is obtained and it is shown experimentally that the cavity length can be four times longer than when using standard NRZ encoding for the same bit-error-rate (BER) performance.

\section{PRINCIPLE AND EXPERIMENTAL SETUP}

The principle of a self-seeded RSOA cavity is as follow. The cavity is based on an RSOA located at the optical network unit (ONU) playing the role of the gain medium and of one of the mirrors of the cavity. The remote node (RN) is composed of a WDM multiplexer that selects the spectral bandwidth in which lasing will occur. The filtered signal is then reflected back to the RSOA after a second mirror, also located at the RN, enabling the establishment of a steady state regime. The cavity length can vary from a few hundreds of meters to a few tens of kilometers, depending on the distribution fiber length between the ONU and the RN. This configuration enables both colorless operation and the sharing of the multiplexer between several customers who can be several kilometers apart. Data are directly applied to the RSOA for upstream communication in PON links. In our experimental setup depicted in Fig. 1, the

A. Maho, R. Brenot are with III-V Lab, F-91461 Marcoussis Cedex, France (e-mail: Romain.Brenot@3-5lab.fr). 
RSOA is based on a quantum-dash active medium with a small signal gain of $25 \mathrm{~dB}$ at $1540 \mathrm{~nm}$, a polarization dependent gain of $20 \mathrm{~dB}$ and a $6-\mathrm{dB}$ electrical bandwidth of $3 \mathrm{GHz}$ at $100-\mathrm{mA}$ bias. The RSOA is directly modulated by the signal to be transmitted, as it will be described later. The association of a $45^{\circ}$ Faraday rotator (FR) at the output of the RSOA (at the $\mathrm{ONU}$ ) with a $90^{\circ}$ Faraday mirror (FM) (at the RN) was shown to guarantee a stable polarization in the RSOA corresponding to its maximum gain [11]. The distribution fiber is a standard single-mode fiber (SSMF) whose length varies from 0 to $4 \mathrm{~km}$. In the RN, the WDM multiplexer is a Gaussian arrayed waveguide grating (AWG) with channel spacing of $100 \mathrm{GHz}$, channel bandwidth of $60 \mathrm{GHz}$ at $3 \mathrm{~dB}$ and insertion loss of $2 \mathrm{~dB}$; the channel at $1542 \mathrm{~nm}$ is used in this experiment. It is followed by a $10 / 90 \%$ optical coupler to extract part of the source signal and a FM with $90 \%$ reflectivity.

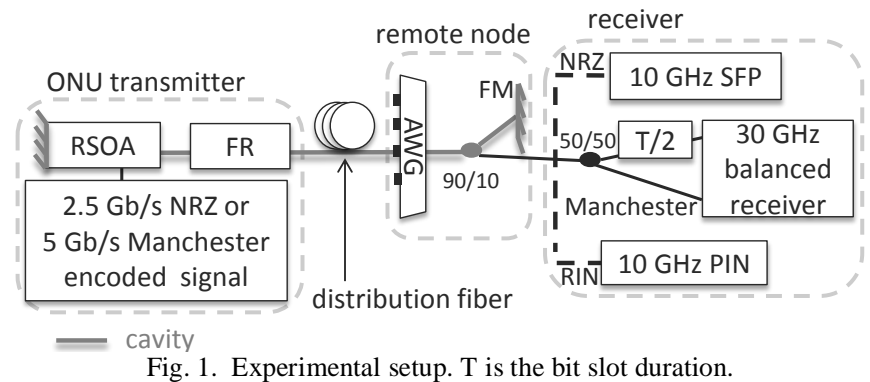

In this work, we compare two different transceivers performance, one with NRZ-encoding and single-ended detection, the other with Manchester encoding and balanced detection. The impact of cavity length is studied and correlated to the RIN of the source. In the classical NRZ encoding case at $2.5 \mathrm{Gbit} / \mathrm{s}$, a $10-\mathrm{GHz}$ PIN photodiode in small form-factor pluggable (SFP) receiver was used.

In the case of Manchester encoding, the signal is modulated at $5 \mathrm{Gbit} / \mathrm{s}$ by a succession of pairs ' 01 ' or ' 10 ', each pair corresponding to one of the two Manchester binary states, thus resulting in the same data rate of $2.5 \mathrm{Gbit} / \mathrm{s}$. In both encoding cases a pseudo-random binary sequence (PRBS) of length $2^{7}-1$ is used. The differential receiver scheme consists in splitting the signal into two paths with a delay of half the bit slot duration (T) before detection in balanced photodiodes [9]. In this work, we used a pair of $30-\mathrm{GHz}$ PIN photodiodes followed by a 6.7-GHz electrical low-pass filter.

Finally, for RIN measurement, a 10-GHz linearly amplified receiver and a 40-GHz electrical spectrum analyzer are used.

\section{RIN MEASUREMENTS}

Figure 2(a) depicts the results of RIN measurements for different cavity lengths. The spectra are measured from $5 \mathrm{MHz}$ to $2.5 \mathrm{GHz}$ with a resolution bandwidth of $3 \mathrm{MHz}$ when a bias current of $150 \mathrm{~mA}$ is applied to the RSOA. It can be observed that the RIN level increases with the cavity length (i.e. distribution fiber length). A large rise of the RIN in the low frequency region of up to $20 \mathrm{~dB} / \mathrm{Hz}$ is notably observed between the 9-m and 4-km long cavities. The origin of this large RIN increase below $20 \mathrm{MHz}$ is still under investigation. It is confirmed by the observation of the oscilloscope traces and intensity histograms in Fig. 2(b), which were obtained for cavity lengths of $1 \mathrm{~km}$ and $4 \mathrm{~km}$ when a direct-current (DC) bias of $150 \mathrm{~mA}$ was applied to the RSOA. In those measurements, it was ensured that the same average optical power of $-3 \mathrm{dBm}$ was incident on the photodiode in both cases so that a direct comparison can be performed. A broader power distribution can be observed in the case of the longer cavity. This RIN increase is likely to be due to chromatic dispersion as reported in [4]. Indeed, it was experimentally observed that, when the distribution fiber consists of dispersion shifted fiber, the power distribution does not evolve with the cavity length whereas the use of SSMF leads to broader power distributions when the cavity length increases, as in our experiment.
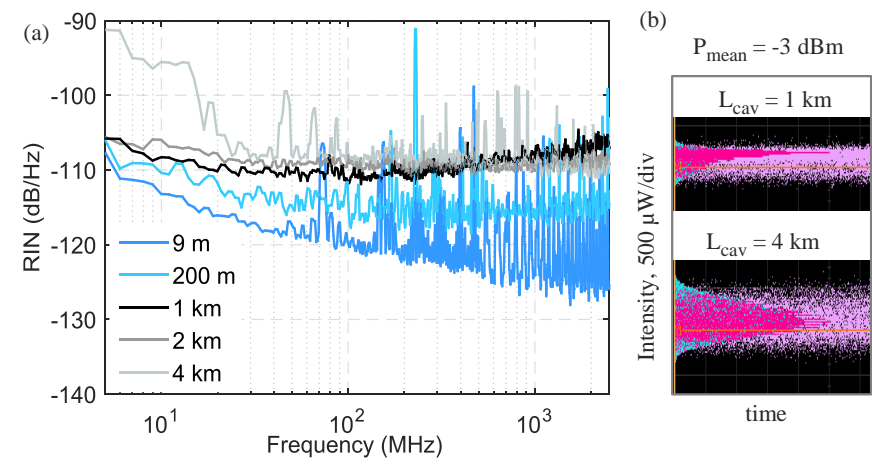

Fig. 2. Evolution of the intensity noise with increased cavity length: (a) RIN measurement, (b) Oscilloscope traces with intensity histograms.

The strong degradation of the laser intensity noise with the cavity length makes the use of such long cavities difficult in conventional systems employing NRZ encoding.

\section{SYSTEM EXPERIMENT}

For this proof-of-concept experiment, the transceiver performance of the uplink employing NRZ encoding is first studied as a function of cavity length. The bit-error-rate increases with the cavity length and an error floor appears at a BER of $10^{-4}$ for a cavity length of $1 \mathrm{~km}$, as shown in Fig. 3. It is then not possible to further increase the cavity length. Eye diagrams for cavity lengths of $9 \mathrm{~m}$ and $1 \mathrm{~km}$, represented as insets in Fig. 3, clearly show the presence of strong amplitude noise for the longer cavity.

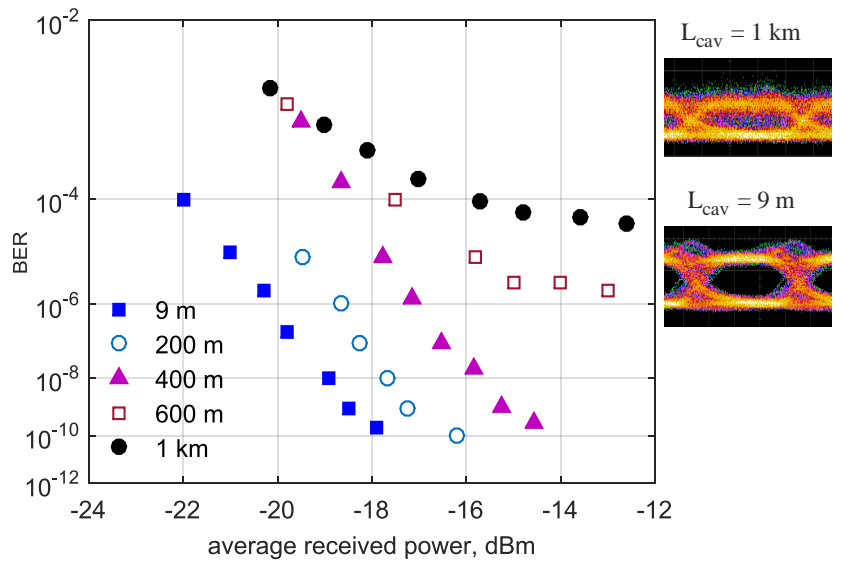

Fig. 3. BER as a function of cavity length for NRZ encoding. The insets represent the eye diagrams for $9-\mathrm{m}$ and $1-\mathrm{km}$ long cavities.

Eye diagrams measurements for Manchester encoding are reported in Fig. 4. The eye diagrams of Fig. 4(a) and (b) are 
measured with a PIN single-ended photodiode with $32-\mathrm{GHz}$ bandwidth whereas the eyes of Fig. 4(c) and (d) are measured using balanced detection with $30-\mathrm{GHz}$ balanced PIN photodiodes. The cavity lengths are $200 \mathrm{~m}$ in Fig 4(a) and (c) and $1 \mathrm{~km}$ in Fig. 4(b) and (d). For both cavity lengths, the incident optical power on the receiver is identical. The observed eye diagram degradation is therefore due to the RIN increase in the cavity. However, if the eye diagrams measured using a single-ended photodiode clearly show an increase of noise with increasing cavity length as for NRZ encoding, the eye diagrams obtained after balanced detection remain less noisy due to the effect of noise subtraction.

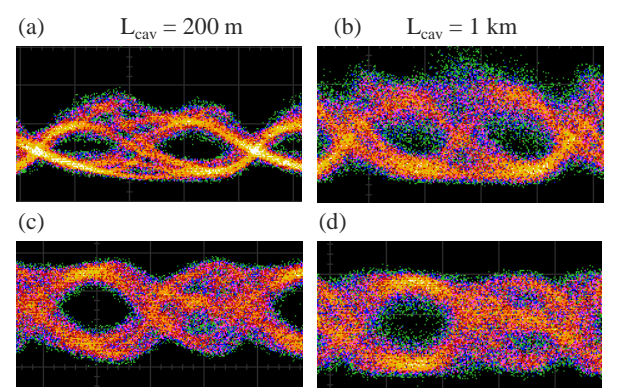

Fig. 4. Eye diagrams for cavity lengths of $200 \mathrm{~m} \mathrm{((a)} \mathrm{and} \mathrm{(c))} \mathrm{and} 1 \mathrm{~km}$ ((b) and (d)) in the case of Manchester encoding using a single photodiode ((a) and (b)) and using a balanced photodiode ((c) and (d)).

Finally, the BER is measured after balanced detection (Fig. 5). In the case of Manchester encoding with balanced detection, a 6-dB power penalty is expected compared to NRZsingle ended receiver in the case of a direct detection configuration limited by thermal noise (i.e. without optical preamplification). Indeed, in the case of balanced detection, the thermal noise contributions from the two photodiodes being independent, the total resulting noise power will double. Moreover, the fact that Manchester encoding requires twice the bandwidth of NRZ results in another doubling of the thermal noise variance. Consequently, at equivalent input average optical power and photodiode thermal noise spectral density, the quality factor of NRZ single-ended detection can be shown to be 4 times the quality factor of Manchester-balanced detection, which would lead to $6 \mathrm{~dB}$ penalty for the latter encoding. This analysis is valid as long as the photodiodes thermal noise is the limiting factor. If the RIN of the transmitter cannot be neglected, a reduction of the difference in sensitivity between the two transceivers is expected. Indeed, the impact of RIN will be reduced when balanced detection is used, whereas it remains more detrimental in the single-ended receiver case.

In this experiment, for a cavity length of $9 \mathrm{~m}$, the penalty between both configurations at $10^{-4}$ is about $5 \mathrm{~dB}$, which is not far from the expected value when thermal noise is dominant. For a cavity length of $1 \mathrm{~km}$, the penalty is reduced to $2 \mathrm{~dB}$ and the error floor is reduced to a BER of $2 \times 10^{-7}$. It can be concluded that the noise variance at the decision circuit is not dominated by the photodiode thermal noise but by the RIN for this cavity length. Finally, for a 4-km long cavity, no transmission is possible in the case of single-ended receiver, whereas the signal is still detected with an error floor of $10^{-4}$ using balanced detection.

It should be noted that the bandwidths of the photodiodes

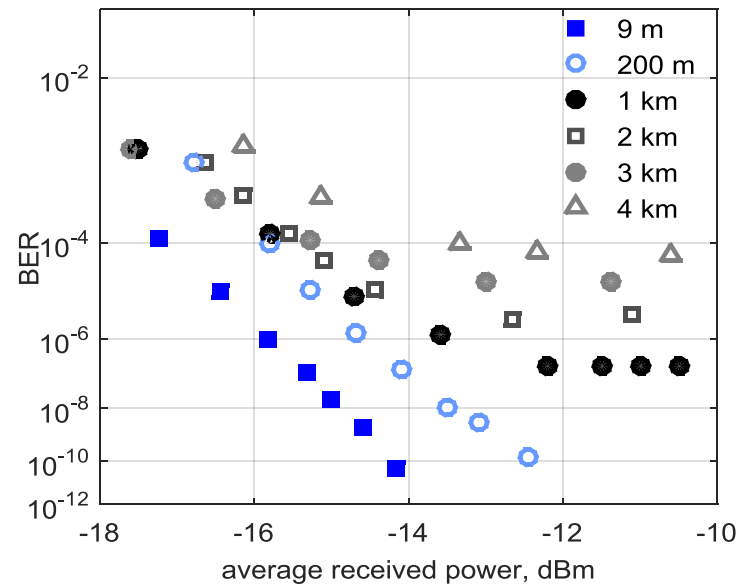

Fig. 5. BER as a function of received power for different cavity lengths in the case of Manchester encoding.

used in this experiment were not fully optimized. Using photodiodes with optimized bandwidths is expected to improve the system performance. Furthermore, in a PON configuration, a feeder fiber of a few tens of kilometers would be used inbetween the transmitter and the central office. The proposed solution remains to be tested in a full transmission experiment.

\section{CONCLUSION}

In this work, we have shown experimentally how to take advantage of Manchester encoding associated to balanced detection in order to obtain a better tolerance to RIN in a selfseeded RSOA fiber cavity laser. Even though an increase of the RIN with cavity length is observed, the better tolerance to intensity noise of the proposed solution allows to multiply by 4 the allowed distance between customer and remote node compared to standard NRZ encoding.

\section{REFERENCES}

[1] E. Wong, K. Lee and T. Anderson, "Directly modulated self-seeding reflective semiconductor optical amplifiers as colorless transmitters in wavelength division multiplexed passive optical networks," J. Lightw. Technol., vol. 25, no. 1, pp. 67-74, 2007.

[2] G. Simon, F. Saliou, P. Chanclou, Q. Deniel, D. Erasme and R. Brenot, "70 km external cavity DWDM sources based on O-band self-seeded RSOAs for transmissions at 2.5Gbit/s," in Opt. Fib. Comm. Conf., San Francisco, California, USA, March 2014, W3G5.

[3] P. Parolari, L. Marazzi, M. Brunero, M. Martinelli, R. Brenot, A. Maho, S. Barbet, G. Gavioli, G. Simon, S. D. Le, F. Saliou, and P. Chanclou "C- and O-band operation of RSOA WDM PON self-seeded transmitters up to $10 \mathrm{~Gb} / \mathrm{s}$," J. Opt. Commun. Netw., vol. 7, no. 2, pp. A249-A255, 2015.

[4] S. A. Gebrewold, L. Marazzi, P. Parolari, R. Brenot, S. P. O. Duill, R. Bonjour, D. Hillerkuss, C. Hafner, and J. Leuthold, "Reflective-SOA fiber cavity laser as directly modulated WDM-PON colorless transmitter," IEEE J. Sel. Topics Quantum Electron., vol. 20, no. 5, pp. 503-511, 2014.

[5] A. Maho, G. Simon, S. Barbet, F. Lelarge, F. Saliou, P. Chanclou, P. Parolari, L. Marazzi, M. Brunero, M. Martinelli, G. Grebewold, J. Leuthold and R. Brenot, "Demystification of the self-seeded WDM access," J. Lightw. Technol., vol. 34, no. 2, pp. 776-782, 2016.

[6] A. Borghesani, I. F. Lealman, A. Poustie, D. W. Smith, and R. Wyatt, "High temperature, colourless operation of a reflective semiconductor optical amplifier for 2.5Gbit/s upstream transmission in a WDM-PON," in Europ. Conf. on Opt. Comm., Berlin, Germany, sept. 2007, We6.4.1.

[7] M. L. Deng, B. Y. Cao, R. P. Giddings, Y. X. Dong, N. Jiang, D. Nesset, K. Qiu and J. M. Tang, "Intra-cavity chromatic dispersion impacts on 10- 
$\mathrm{Gb} / \mathrm{s}$ optical OFDM transmissions over 25-km dual-RSOA-based selfseeded PON systems," IEEE Photon. J., vol. 7, p. 7200112, 2015.

[8] A. Joshi, X. Wang, D. Mohr, D. Becker and C. Wree, "Balanced photoreceivers for analog and digital fiber optic communications," in Proc. SPIE 5814, Enabling Photonics Technologies for Defense, Security, and Aerospace Applications conf., Orlando, Florida, USA, May 2005.

[9] M. E. Chaibi, L. Bramerie, S. Lobo and C. Peucheret, "Mitigation of mode partition noise in quantum-dash Fabry-Perot mode-locked lasers using Manchester encoding," in Europ. Conf. on Opt. Comm., Düsseldorf, Germany, 2016, Tu.2.E.6.

[10] Y. Yamada, Y. Shibata, T. Okugawa and K. Habara, "Tolerance for optical coherent crosstalk of a novel Manchester-code receiver," in Europ. Conf. on Opt. Comm., Madrid, Spain, 1998, vol. 1, p. 61.

[11] M. Martinelli, L. Marazzi, P. Parolari, M. Brunero and G. Gavioli, "Polarization in retracing circuits for WDM-PON," IEEE Photon. Technol. Lett., vol. 24, no. 14, pp. 1191-1193, 2012. 